\title{
On the multiplicity and symmetry of particle attractors in confined non-isothermal fluids subjected to inclined vibrations
}

\author{
Marcello Lappa ${ }^{1}$ \\ ${ }^{1}$ Department of Mechanical and Aerospace Engineering, University of Strathclyde \\ James Weir Building, 75 Montrose Street, Glasgow, G1 1XJ, UK \\ email: marcello.lappa@strath.ac.uk - marlappa@unina.it
}

\begin{abstract}
We investigate the relationship between the motion of solid particles and related clustering dynamics under the combined influence of a large-scale convective flow and vibrationsinduced small-scale displacements in microgravity conditions. Such a study is motivated by the relatively recent discovery of a new class of particle attractors for which thermal and inertial effects play a fundamental role whereas particle-to-particle interactions are not essential. The key aim is to expand the set of predictive links between flow properties and particle behavior identified in the author's earlier work (Phys. Fluids, 26(9), 093301, 2014). Insights are sought from consideration of two archetypal settings, both originating from the classical problem of a differentially heated cubic cavity, which in one case is subjected to vibrations contained in a plane parallel to one of the insulated walls (i.e. having a component in the direction parallel to the temperature gradient) and in the other experiences periodic accelerations arbitrarily oriented in space. The present study reveals that the multiplicity of the particle attractors, previously thought to be a universal number $(\mathrm{N}=2$ according to earlier studies), is not a constant. The two distinct particle attractors seen in past investigations can undergo "coalescence" in some cases and exist as a single attractor over a relatively wide range of conditions. Moreover, in addition to the ellipsoid, cylinders and conical surfaces, other shapes of the attractors become possible when the vibrations are inclined with respect to the adiabatic walls, such as amphora-like or saddle-horse paraboloid structures.
\end{abstract}

Keywords: Particle segregation, thermovibrational flow, inclined vibrations.

\section{Introduction}

Two-phase dispersive flows are prevalent in science and widely studied in areas such as engineering, electronics, physics, life sciences, chemistry, materials science and even astrophysics (see, e.g., Lappa, 2016a and references therein).

In the majority of situations, a proper simulation of the related dynamics requires proper modeling of the flowing particulate media at distinct spatial and temporal scales, especially when the "carrier" flow (the flow transporting the solid phase) experiences disturbances which make it irregular or 
time-dependent (Houghton, 1961; Hjelmfelt and Mockros, 1966; Mei et al., 1991; Maxey et al., 1997; Coimbra and Rangel, 2001; Lappa and Carotenuto, 2003; Coimbra et al., 2004). Many of these analyses have shown that there are contingencies in which the distribution of solid particles tends to acquire a recognizable level of self-organization (i.e. a precise morphology and/or topology in space and/or lines of evolution in time). Moreover, in general, there exist, between the limiting purely regular and irregular solid-phase distributions in space (and/or time), numerous intermediate situations. One of the most remarkable achievements obtained in recent years is the discovery that the exact path the system takes in its evolutionary progress towards one of these situations depends essentially on its nature being reversible or dissipative.

While an initially uniform distribution of passive tracers, in principle, would remain uniform as time passes, circumstances can be identified for which particles produce strong inhomogeineities, such as formation of clusters. This accumulation is due to the convergence of particle trajectories onto certain sets in the position-velocity phase space, which are called "attractors" using a terminology borrowed from the general field concerned with the study of non-linear systems. Such a process can be promoted by different (non-reversible) physical effects ranging from electrostatic phenomena (Castellanos, 2005; Love et al., 2014) and hydrodynamic (particle-to-particle) interactions (Langbein, 1991; Ivanova et al., 2005; Kozlov et al., 2006) to the intrinsic dissipative nature of "inertial" particles. All such effects can induce particle displacement, causing a departure of particles from the paths that would be ideally taken by ideal mass-less and point-source tracers not subjected to any surface or body force.

In the case of inertial particles, surface forces (of an electrostatic or hydrodynamic nature) are not necessary, with recognizable particle structures being essentially a consequence of the finiteness of mass and size of each isolated particle. This specific category of attractors has been approached by different directions and different research groups with various background and perspectives. This synergy has led over the years to establishing a common elegant theoretical framework that is now generally referred to as the study of inertial particle dynamics.

Much work has been devoted to the investigation of such phenomena when the carrier flow has a relatively strong turbulent behavior for which particle structures typically emerge in the form of fractal aggregates (in flow regions of relatively high enstrophy or vorticity depending on the particle-to-fluid density ratio, see, e.g., Tagawa et al., 2012 and references therein) or cluster onto a network of "caustic" lines (analogous to the pattern of refracted light that can be seen on the bottom of a swimming pool, Wilkinson et al., 2007). Most recently, however, a new line of inquiry has been originated by the remarkable discovery that much more regular structures can be formed if the carrier flow satisfies certain requirements. It has been understood that such phenomena occur when relatively simple (in terms of frequency spectrum) flows are produced by fluid-dynamic instabilities (e.g., Hopf bifurcations) such as those typically occurring in a variety of thermal flows of natural origin (e.g., thermogravitational or thermocapillary flows), which are initially steady, and become oscillatory in time when the related control parameter exceeds a given threshold. This has stimulated a number of publications (Schwabe et al., 2006; Ueno et al., 2008; Schwabe and Mizev, 
2011; Pushkin et al., 2011; Melnikov et al., 2013; Gotoda et al., 2015 and 2016). Such flows can be still considered "laminar" and the associated dynamics "deterministic" under a certain perspective. Alternatively, similar phenomena can be promoted by the application of an external time-varying (periodic) "forcing" to a non-isothermal system (leading to the so-called forced "thermovibrational convection").

While in the first situation, particle preferential segregation can be explained in terms of a "phase locking" process between a travelling fluid-dynamic disturbance (a wave) and the typical frequency of motion or rotation of a generic particle (as a result of which, the motion of an individual particle in the three-dimensional flow gets synchronized with the wave, Pushkin et al., 2011), in the latter case particle self-assembly is due to the delicate interplay (yet a kind of "synchronization") between particle inertial motion and oscillatory thermovibrational effects, which tends to constrain particles to specific regions of the physical space.

The subsets of space where such phenomena occur evolve accordingly from perfect onedimensional (1D) structures with particles orderly aligned along a closed helical curve or circuit seen in the case of a single travelling wave (Schwabe et al., 2006; Ueno et al., 2008; Lappa, 2013a,b), or planar (2D) areas for the case of two waves counter-propagating with equal amplitude (Schwabe and Mizev, 2011; Lappa, 2014a), to the three-dimensional (3D) geometric objects resembling the typical "quadrics" of projective geometry (open or compact surfaces such as cylinders, ellipsoids and conical shapes, etc.), which emerge when single-wave or multi-wave flow patterns are replaced by vibrations and related thermal (buoyancy) effects (Lappa, 2014b).

Though the particle structures revealed by these studies might be the signature of some hidden analytic order underlying the formation and existence of such attractors, the related cause-and-effect relationships have not been clarified yet. There are still many unresolved challenges faced by researchers in these fields which emphasize the need to develop new and to enhance the existing theoretical frameworks.

Continuing a line of research undertaken in earlier works, here we consider the interplay between the mechanisms of solid particle spontaneous accumulation and ordering in the presence of "translational vibrations" (g-jitters). In this regard the present study may be regarded as an extension of Lappa (2014b). While in that work we contented ourselves with developing the subject solely for the case of vibrations perpendicular to the imposed temperature gradient, here related directions can form an arbitrary angle in space.

In practice, the present analysis stems from the realization that since the effect of an inclined body force on a non-isothermal fluid system is expected to display notable differences with respect to standard cases for which gravity is parallel or perpendicular to the temperature difference (Lappa, $2007 \mathrm{ab}$ and 2011), similar dramatic changes may be reflected in the morphology and topology of the corresponding particle "attractors".

Standard buoyancy convection in system with inclined gravity or inclined temperature gradient has been considered by several researchers (Chen and Pearlstein, 1989; Fujimura and Kelly, 1993; Nield, 1994; Busse and Clever, 1992, 2000; Delgado-Buscalioni et al., 1998, 2001; Delgado- 
Buscalioni and Crespo del Arco, 1999 and 2001; just to cite some relatively recent contributions). In comparison to the case of steady gravity, efforts related to the study of thermovibrational convection (i.e. flow induced in the presence of temperature gradients by time-varying accelerations arbitrarily oriented in space) seem to be much more rare and sparse (Demin et al., 1996; Gershuni and Demin, 1998; Shklyaev, 2001; Smorodin, 2003; Demin, 2005).

Starting from the realization that classical methods for the control of two-phase dispersive flows require further developments in order to satisfy the increasing needs of modern application-driven technologies, the final scope of the present study (and associated overarching line of inquiry) is the development of new ideas by which the properties of a dispersed particle-liquid system could be predicted or controlled "a priori" through the use of vibrations. Although this research is too earlystage to attract industrial interest just now, the related results might be regarded as another worthy example of new knowledge relating to the aforementioned "deterministic hydrodynamics" concepts (i.e. innovative approaches taking advantage of fluid-dynamic scenario in which the considered flow displays a naturally-emerging or induced-by-imposed-forcing time-periodic pattern, see, e.g., the recent work by Kozlov et al., 2017).

\section{Mathematical model}

For a proper introduction of governing principles, related mathematical models and balance equations, we consider the case of incompressible fluid undergoing harmonic motions around a fixed direction ( $\underline{\hat{n}}$ being the related unit vector) in microgravity conditions.

\subsection{Vibrations}

The specificity of the considered vibrations is that their linear effects have zero-time averaged value. Hence, only nonlinear effects are expected to play a role even if they are small compared to the instantaneous linear effects. Such vibrations are modelled here by assuming a displacement of the considered geometrical system along a given direction ( $\underline{\hat{n}}$ is the related unit vector) varying in time as $\underline{s}^{l a b}(t)=b \sin (\omega t) \underline{\hat{n}}$ where $\mathrm{b}$ is the amplitude and $\omega=2 \pi f$ ( $f$ is the frequency). Such a displacement, in turn, implies a "gravity modulation" or g-jitter (Monti et al., 1998 and 2001) $\underline{g}(t)=g_{\omega} \sin (\omega \mathrm{t}) \underline{\hat{n}}$ where $g_{\omega}=\mathrm{b} \omega^{2}$. Such inertial perturbations are known to excite the response of "inhomogeineities" present in the considered fluid system. These can be due (see, e.g., Lappa, 2013c) to the presence of dispersed matter such as minute hard spheres, or to density inhomogeineities, as those induced in a simple fluid by temperature gradients (which lead to the socalled thermovibrational flow, Savino and Lappa, 2003, Lappa, 2016b, etc.). The related principles and governing equations are illustrated in the following sections. 


\subsection{The Geometry}

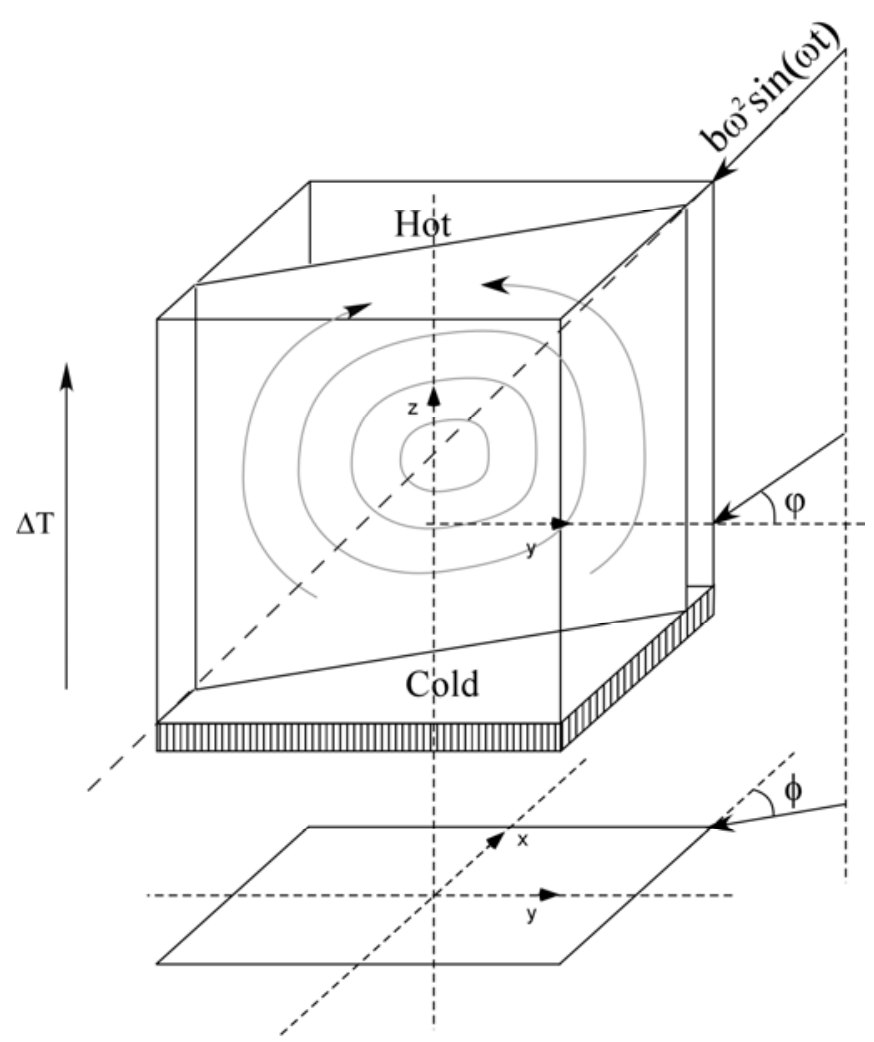

Figure 1: The periodic acceleration vector has three distinct components along the coordinate axes (the angles formed with the $\mathrm{y}$ axis and the $\mathrm{x}$ axis by its projections in the $\mathrm{yz}$ and $\mathrm{xy}$ planes are denoted by $\varphi$ and $\phi$, respectively. The wall located $z=0.5$ is assumed to be heated, the other at $z=-$ 0.5 cooled with adiabatic conditions on the remaining sidewalls.

As in Lappa (2014b), we consider a cubic cavity with characteristic size L (Fig.1). The cube box should indeed be regarded as the most suitable "archetypal setting" for the investigation of such phenomena (it is a geometrical symmetric system by which one is allowed to explore the fundamental properties of the particle attractors when one of these symmetries is broken).

TABLE I: Liquid-solid system properties

\begin{tabular}{ll}
\hline \hline Fluid $\left(\mathrm{NaNO}_{3}\right)$ density $\rho, \mathrm{kg} \mathrm{m}^{-3}$ & 1904 \\
Kinematic viscosity $\mathrm{v}, \mathrm{m}^{2} \mathrm{~s}^{-1}$ & $1.27 \times 10^{-6}$ \\
Thermal diffusivity $\alpha, \mathrm{m}^{2} \mathrm{~s}^{-1}$ & $1.58 \times 10^{-7}$ \\
Thermal expansion coefficient $\beta_{\mathrm{T}}, \mathrm{K}^{-1}$ & $1.25 \times 10^{-3}$ \\
Particle/liquid density ratio $\xi$ & 1.85 \\
\hline \hline
\end{tabular}


The value of the Prandtl number is $\operatorname{Pr}=8(\operatorname{Pr}=v / \alpha$ where $v$ is the fluid kinematic viscosity and $\alpha$ its thermal diffusivity) corresponding to $\mathrm{NaNO}_{3}$. The properties of the considered solid-liquid system are summarized in Table I. Sodium nitrate has enjoyed widespread consideration in past works dealing with thermal convection because of its relevance to many materials science problems (especially the production of transparent crystals for optical and optoelectronic applications, Lan and Kou, 1991, see also Melnikov et al., 2013).

As shown in Fig. 1, the periodic-acceleration vector has three distinct components along the coordinate axes (the angles formed with the horizontal direction and the $\mathrm{x}$ axis by its projections in the yz and xy planes are denoted here by $\varphi$ and $\phi$, respectively).

Briefly stated, the problem is to determine how the combined action exerted on particles by the large-scale flow of thermovibrational nature and the time-varying body force (acting on a small scale directly on particles) can determine specific patterns of dispersed solid matter when the angles $\varphi$ and $\phi$ are varied in relatively wide ranges.

As in earlier works, the models used here rest on the theory of partial differential equations. Such equations take a different form according to the category of phenomena considered, these being the liquid large-scale flow (Sect. 2.3) or the motion of the dispersed solid particles (Sect. 2.4).

\subsection{Nondimensional balance equations for the liquid phase}

Using the canonical Boussinesq approximation to account for the presence of body accelerations as defined in Sect. 2.1 and conventional scalings to put the equation in non-dimensional form (scaling distances by the system extension $\mathrm{L}$, the velocity components by the energy diffusion velocity $\mathrm{V}_{\alpha}=$ $\alpha / \mathrm{L}$, time and pressure by $\mathrm{L}^{2} / \alpha$ and $\left.\rho \alpha^{2} / \mathrm{L}^{2}\right)$, the continuity and momentum equations simply read:

$\underline{\nabla} \cdot \underline{\mathrm{V}}=0$

$\frac{\partial \underline{V}}{\partial}=-\underline{\nabla} p-\underline{\nabla} \cdot[\underline{V} \underline{V}]+\operatorname{Pr} \nabla^{2} \underline{V}+\operatorname{Pr} R a_{\omega} T \sin (\varpi t) \underline{\hat{n}}$

where $\underline{V}$ is the fluid velocity and $\mathrm{p}$ its pressure. Moreover, $R a_{\omega}=\frac{b \omega^{2} \beta_{T} \Delta T L^{3}}{v \alpha}$ (where $\beta_{\mathrm{T}}$ is the thermal expansion coefficient) can be regarded as a variant of the classical Rayleigh number with the steady acceleration being replaced by the amplitude of the considered monochromatic periodic acceleration. In this form the degrees of freedom of the problem are reduced to four independent nondimensional parameters only: the Prandtl number, the nondimensional frequency $(\varpi)$, the nondimensional acceleration amplitude $(\gamma)$ and the buoyancy factor $(\beta)$ defined as:

$\varpi=\frac{\omega L^{2}}{\alpha}, \quad \gamma=\frac{b \omega^{2} L^{3}}{\alpha^{2}}$ and $\quad \beta=\left(\beta_{T} \Delta T\right)$

where, obviously $\operatorname{Ra}_{\omega}=\gamma \beta / \operatorname{Pr}$. 
The presence of effects of thermovibrational nature obviously requires consideration of the energy balance equation, which, introducing the nondimensional temperature as $\mathrm{T}=\left(\overline{\mathrm{T}}-\overline{\mathrm{T}}_{\mathrm{m}}\right) / \Delta \mathrm{T}$ (where $\Delta \mathrm{T}$ is the imposed temperature difference and $\overline{\mathrm{T}}_{\mathrm{m}}$ a reference dimensional value), reads:

$$
\frac{\partial T}{\partial t}+\underline{\nabla} \cdot[\underline{V} T]=\nabla^{2} T
$$

The kinematic conditions to be imposed on the walls simply reflect the well-known no-slip and impermeability properties of solid boundaries $(\underline{V}=0)$. For problem closure, such conditions, have to be supplemented with those for the energy equation. As anticipated in Sect. 2.2, the lower $(\mathrm{z}=-0.5)$ and upper $(\mathrm{z}=0.5)$ walls of the domain are assumed to be at uniform and constant (nondimensional) temperatures $\mathrm{T}_{0}=0$ and $\mathrm{T}_{1}=1$ respectively, while the sidewalls are adiabatic, i.e. the $\partial T / \partial \mathrm{n}=0$ condition is imposed there. As in Lappa (2014b), at the initial instant $(\mathrm{t}=0)$, a linear temperature distribution along the $\mathrm{z}$ axis is considered, i.e. $T=\mathrm{z}+1 / 2$, while the fluid is assumed to be in quiescent conditions (i.e. $\underline{V}=0$ everywhere).

\subsection{The Dispersed Phase}

The particles are assumed to have a density greater than that of the carrier medium. The method used to track the generic particle position pertains to a group comprising numerical techniques that are based on the Lagrangian trajectory description of the dispersed phase. In other words, these methods solve the traditional balance equations for energy and momentum over the entire physical space considered (the cubic geometry) and then use a specific particle tracking equation to determine the trajectories of individual particles. Such a specific equation is linked to the first group of equations by the fact that the bulk solutions of the liquid-phase balance equations have to be used to properly reconstruct (via interpolation between the particle instantaneous position and the fixed Eulerian grid nodes) the inertial and drag forces acting on the generic solid particle. The flexible and modular nature of the resulting approach allows both macroscopic information (i.e. the macrophysical problem related to the overall solid-liquid pattern formation) and microscopic details (i.e. the microphysical problem related to the motion of each individual particle) to be captured in a single numerical treatment.

Some proper assumptions, obviously, must be invoked, to make such modus operandi effective. As indicated above, the resulting algorithm does not consider the feedback action (the modulation effect) of particles on the liquid flow. The latter clause means that flow characteristics can be taken as pre-determined by solution of eqs. (1-4) only and, therefore, are unaffected by the presence of the disperse phase. In practice, this makes sense under the assumption that the concentration of the dispersed phase in the flow is small, i.e. that the system is dilute (Langbein, 1991; Ellison et al., 1995; Thomson et al., 1997; Kozlov et al., 2006; Hassan et al., 2006; Lappa, 2013c). The 
appropriate equation taking into account vibrations, particle inertia and viscous drag forces (see, e.g., Kuhlmann et al., 2014 and references therein), accordingly, reads:

$$
\frac{d \underline{V}_{\text {partcl }}}{d t}=\frac{1}{\xi+1 / 2}\left[-\frac{\operatorname{Pr}}{S t}\left(\underline{V}_{\text {partcl }}-\underline{V}\right)+\frac{3}{2} \frac{d \underline{V}}{d t}+\frac{3}{2}(\underline{V} \cdot \underline{\nabla} \underline{V})\right]+\frac{\xi-1}{\xi+1 / 2} \gamma \sin (\varpi t) \underline{\hat{n}}
$$

where $\underline{V}_{\text {part }}\left(\mathrm{u}_{\text {part }}, \mathrm{v}_{\text {part }}, \mathrm{w}_{\text {part }}\right)$ and $\underline{V}$ are the particle and fluid velocity, respectively. This equation includes the added mass effect, as witnessed by the presence of the coefficient " $3 / 2$ " in front of the terms corresponding to the fluid substantial derivative and the division by $\xi+1 / 2$ applied to the entire right hand side of the equation (we do not consider the Basset force as according to the studies by Coimbra and Rangel, 2001 its influence is negligible for $\varpi<5 \times 10^{3}$, the reader being also referred to Lappa, 2013c for additional details and a review study of the literature in this regard). Nondimensional parameters relevant to eq. (5) are $\xi=\rho_{\mathrm{P}} / \rho$, the ratio of the particle to the fluid density, the particle Stokes number, defined a $\mathrm{St}=\tau v / \mathrm{L}^{2}$ (Melnikov et al., 2013; Kuhlmann et al., 2014) where $\tau$ is the so-called particle relaxation time ( $\tau=2 R^{2} / 9 v$, R being the particle radius), i.e.

$S t=\frac{2}{9} \frac{R^{2}}{L^{2}}=\frac{\operatorname{Pr} \eta}{\xi-1}$

or its related parameter $\eta=(\xi-1) \operatorname{St} / \operatorname{Pr}$ (accounting for the combined effect of particle inertia and drag, Pushkin et al., 2011; Lappa, 2013a,b). Following Hjelmfelt and Mockros (1996) and Coimbra and Rangel (2001), we also define the so-called shear-rate based (or "vibrational") Stokes number for the motion of spherical particles in harmonic flows, which for the present conditions can be written as:

$S t_{\omega}=\frac{\rho_{P}}{\rho} \frac{\tau \omega}{2 \pi}=\frac{\xi}{9 \pi} \frac{\varpi S t}{\operatorname{Pr}}=\frac{\xi}{\xi-1} \frac{\eta \varpi}{9 \pi}$

These parameters can be used to determine the transition from the viscous to the inertial regime (see, e.g., Trulsson et al., 2012 and reference therein). According to their value, a suspension can generally be categorized into two classes: Stokesian dynamics for a sufficiently small Stokes number (i.e. dominant viscous forces) and granular suspensions (i.e. where particle inertia is dominant). For the present conditions both $\mathrm{St}$ and $\mathrm{St}_{\omega}$ are $<1$, which justifies our choice to model particle drag as a purely Stokesian drag in eq. (5). Nevertheless, we will still refer to particle dynamics as "inertial" because of the peculiar phenomena under analysis: indeed, if the particles were assumed to have no mass and no size (i.e. ideal point weight-less tracers), though $\mathrm{St}<<1$ none of the phenomena illustrated in the present paper would occur. Some numerical experiments along these lines have been reported in two earlier papers (see Fig. 6 in Lappa, 2014b and Fig. 19 in Lappa, 2016c). If the density of the particles is set equal to the density of the liquid, no particle attractors exist. Particles would remain uniformly distributed in the fluid. 


\subsection{Solution Method and Validation Study}

The governing equations (1-4) have been solved in their complete non-linear and time-dependent form using a projection method, that is an approach by which the computation of the velocity field is articulated into two main macro steps providing separately an intermediate field that has the correct content of vorticity but does not satisfy the continuity equation and a final velocity field, which has both the correct values of divergence and curl. The method has been implemented on a structured Cartesian mesh with staggered collocation of fluid-dynamic variables, using forward differences in time and central-differencing (second order accurate) schemes in space (e.g., Fletcher, 1991). The part concerning the computation of dispersed solid-matter motion (with equations (5)) was validated in earlier studies of the present author (Lappa, 2013c). An exhaustive mesh sensitivity analysis for the coupled system of Eulerian and Lagrangian equations has been reported in Lappa (2016c).

\section{Results and discussion}

The following values of characteristic nondimensional parameters are considered: $\varpi=10^{3}$, $\gamma=1.5 \times 10^{6}$ which correspond to $\operatorname{Ra}_{\omega}=1.58 \times 10^{4}$. This is also equivalent to a value of the so-called Gershuni number:

$G s=\frac{\left(b \omega \beta_{T} \Delta T L\right)^{2}}{2 v \alpha}=\frac{\left(\beta_{T} \Delta T L\right)^{2}}{2 v \alpha}\left(\frac{g_{\omega}}{\omega}\right)^{2}=\frac{\varpi^{2} \Lambda^{2}}{2 \operatorname{Pr}}=\frac{1}{2 \operatorname{Pr}}\left(\frac{\beta \gamma}{\varpi}\right)^{2}=10^{3}$

Such a parameter (which, in general, accounts for the intensity of time-averaged convection, see, e.g., Savino and Lappa, 2003 and Lappa, 2013c), is fixed to Gs $=10^{3}$.

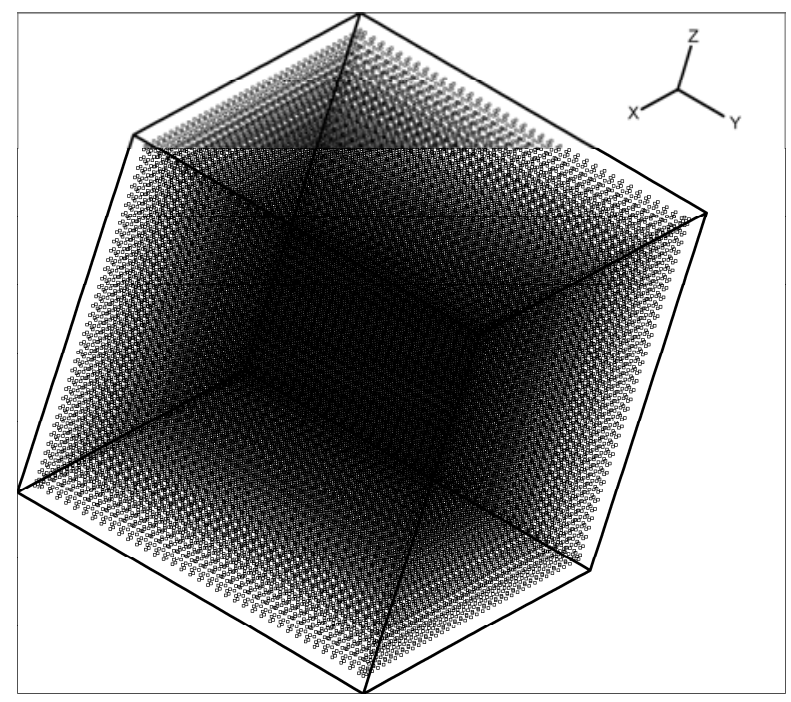

Figure 2: Initial distribution of particles 
As an example, assuming as extension of the enclosure $\mathrm{L}=2 \times 10^{-2} \mathrm{~m}$, the above parameters would correspond to an imposed temperature difference of $67 \mathrm{~K}$ (Lappa, 2014b).

While in earlier works the focus was essentially on assessing the system response and its sensitivity to particle inertia $(\eta)$ and vibration properties $(\varpi)$ keeping fixed the direction of shaking with respect to the imposed temperature gradient $(\varphi=0)$, here, we explore other degrees of freedom of the problem by allowing both angles $\varphi$ and $\phi$ to change over relatively wide intervals (maintaining unchanged the other problem parameters). A proper choice of such quantities is not as straightforward as one would imagine. The formation of the particle structures is prevented if the particle inertia is excessive or it takes a time that tends to infinite in the limit as $\eta \rightarrow 0$. Here we consider $\xi=1.85$ and $\eta=10^{-4}$ (this value of $\eta$ being a good compromise between the two needs of shortening the otherwise computationally prohibitive time required for the formation of welldefined patterns in 3D and of not exceeding the threshold in terms of particle size beyond which such patterns are no longer formed, Lappa, 2014b). To track the system evolution, in particular, solid particles $\left(\cong 6 \times 10^{4}\right)$ are seeded uniformly into the computational domain assuming their velocity equal to zero (Fig. 2). According to a grid-refinement study carried out "a priori" (Lappa, 2014b and $2016 \mathrm{c}$ for related details) a mesh $42 \times 42 \times 42$ has been deemed sufficient to guarantee solution mesh-independence for such conditions.

\subsection{Particle aggregates for $\phi=0$}

With the express intention to introduce concepts and arguments of increasing complexity as the discussion proceeds, in this section we start from the simplest case, namely the situation in which the imposed vibrations are contained in a plane parallel to one of the insulated walls ( $\phi=0$, i.e. vibrations in the xz plane, see Fig. 1). The angle $\varphi$, in particular, is allowed to span the range $0^{\circ} \leq \varphi \leq 90^{\circ}$, the two extremes of this interval representing the limiting situations in which vibrations are exactly perpendicular and parallel to the imposed temperature gradient, respectively.

In the latter case $\left(\varphi=90^{\circ}\right)$, it is known that no thermovibrational convection can arise unless $\operatorname{Ra}_{\omega}$ is larger than a given threshold value depending on the nondimensional frequency $\varpi$. As an example of existing studies on this subject, the reader may consider Hirata et al. (2001), who investigated numerically the canonical reference case of a 2D square cell in microgravity conditions. Assuming a liquid with $\mathrm{Pr}=7$ that is very close to the Prandtl number assumed in the present work, they found the threshold for the onset of convection of vibrational nature in the case of gjitters parallel to the imposed temperature gradient to increase dramatically for increasing values of $\varpi$ (similar results were also obtained by Biringen and Danabasoglu, 1990). In particular, the value of $\mathrm{Ra}_{\omega}$ required for the onset of periodic convection was found to be $>10^{5}$ for $\varpi=10^{3}$, which is an order of magnitude larger than the value of the Rayleigh number considered here $\left(\operatorname{Ra}_{\omega}=\mathrm{O}\left(10^{4}\right)\right)$. Therefore, in the present work the condition $\varphi=90^{\circ}$ may be regarded as a special situation where neither convective states, nor specific particle aggregates can emerge (hereafter we will refer to it as "degenerate state"). 
However, even a small deviation from such a degenerate case can lead to convection, as witnessed by the relatively small (nevertheless appreciable) departure of the velocity field from the purely quiescent conditions for $\varphi=85^{\circ}$. As evident in Fig. 3, in general, a decrease in $\varphi$ leads to an increase in the amplitude of the oscillatory velocity, which attains a maximum in the limit case $\varphi=0$ (i.e. when vibrations are exactly perpendicular to the imposed temperature gradient) and tends to zero in the opposite limit as $\varphi \rightarrow 90^{\circ}$.

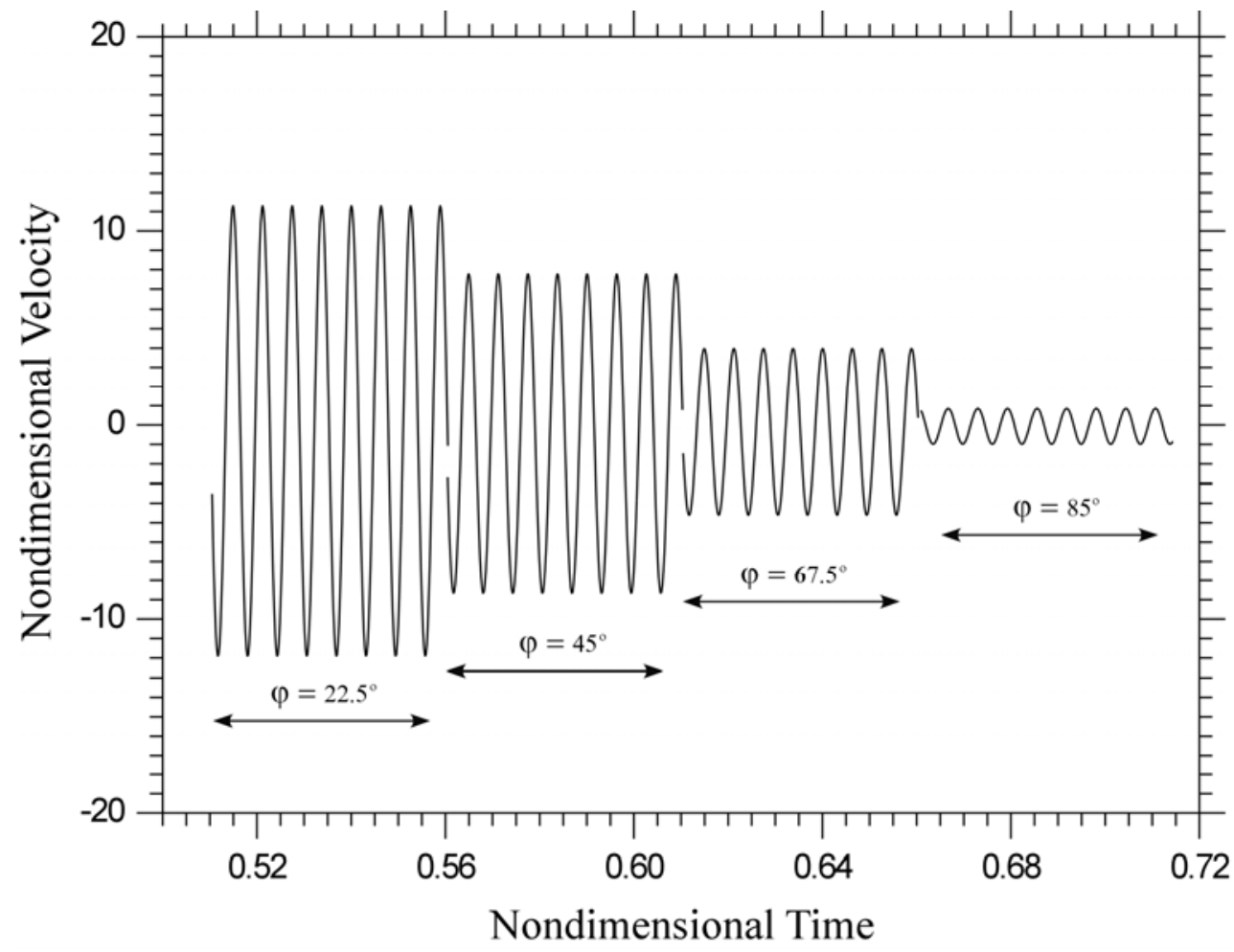

Figure 3: Velocity at a fixed point $(x=0.75, y=0, z=0.75)$ as a function of time for different values of the $\varphi$ angle $\left(\phi=0, \varpi=1 \times 10^{3}, \gamma=1.5 \times 10^{6}, \gamma / \varpi=1.5 \times 10^{3}, \mathrm{Gs}=10^{3}, \mathrm{Ra}_{\omega}=1.58 \times 10^{4}\right)$

Following up on the previous point, additional illuminating insights into the considered flow $\left(\varpi=\mathrm{O}\left(10^{3}\right), \mathrm{Gs}=\mathrm{O}\left(10^{3}\right)\right)$ can be obtained by taking a close look at the mean value displayed by the velocity signals (Fig. 3). The realization that the signals have no average value (or if it exists it is very small as also witnessed by the mirror symmetry of positive and negative peaks), indeed, can be regarded as a clear indication that for the considered conditions the time-periodic nature of the velocity field established inside the cavity is the direct consequence of the interplay between the temperature distribution (which is very close to purely diffusive conditions along the $\mathrm{z}$ axis given the relatively small value of the Gershuni number) and the instantaneous buoyancy effect (originating from the vibration-induced acceleration acting along the direction perpendicular to the effective temperature gradient, i.e. the $\mathrm{x}$ axis direction). 
Stripped to its basics, the mechanism is the following: Vibration-induced buoyancy (via the horizontal acceleration component $\mathrm{b} \omega^{2} \cos (\varphi)$ ) and the vertically stratified temperature distribution in the enclosure will alternatively cooperate to produce a clockwise or anticlockwise convective cell over a cycle of the modulation as shown in Fig. 4. More precisely, in that part of the cycle where the acceleration component in the $\mathrm{x}$ direction is directed along the positive $\mathrm{x}$ axis, the resulting convective cell visible in the $\mathrm{xz}$ plane will be anticlockwise oriented, vice versa clockwise oriented roll when such a component changes its sign.

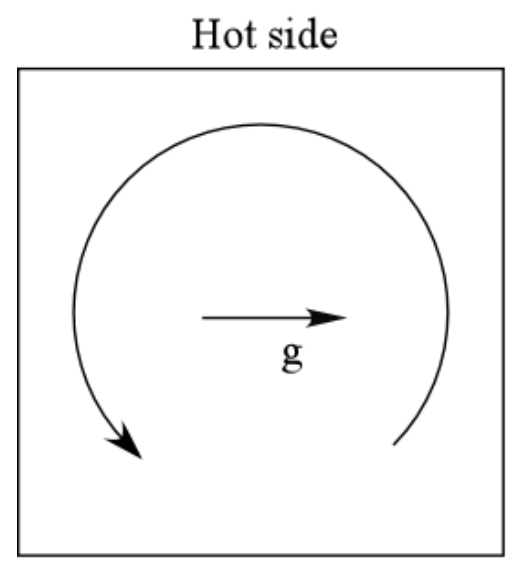

Cold side

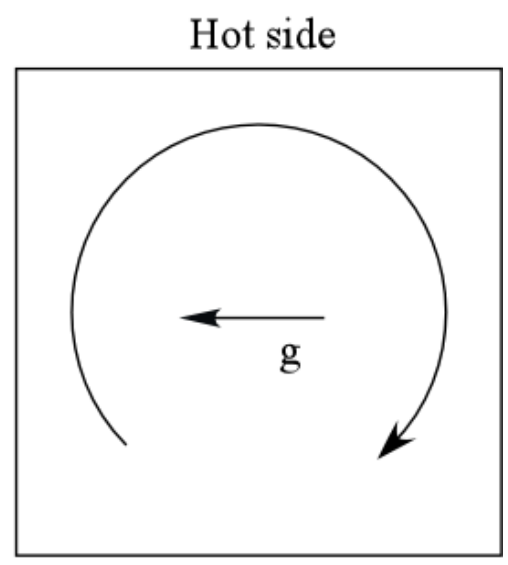

Cold side

Figure 4: Sketch of alternating instantaneous flow modes that are excited in the xz plane when the system is temporarily subjected to an acceleration component acting along the positive (left) or negative (right) $\mathrm{x}$ direction.

Such a perfect symmetry with respect to both space and time, resulting in the periodic replacement of the flow main roll with its mirror image (hereafter simply referred to as "spatio-temporal reversibility"), is a necessary prerequisite for the existence of this class of particle attractors. It would be broken for larger values of the Gershuni number (due to the well-known emergence of significant time-averaged effects, see, e.g., Savino and Lappa, 2003). Such a peculiar condition is obviously retained for different values of the inclination angle, since, as explained before, the component of acceleration parallel to the temperature gradient is not able to induce steady convection that would break the time-reversibility of the flow (Hirata et al., 2001).

Remarkably, however, the spatio-temporal reversibility with respect to the $\mathrm{z}$ direction does not hold for the particles and related motion. Particles are subjected to displacements along both the $\mathrm{x}$ and $\mathrm{z}$ axes due to the two distinct components of the acceleration $\mathrm{b} \omega^{2} \cos (\varphi)$ and $\mathrm{b} \omega^{2} \sin (\varphi)$, respectively, which need no threshold to be exceeded to be effective and, therefore, influence the dynamics in every circumstance.

It is by virtue of such delicate interplay between symmetric and asymmetric effects (namely convective and inertial factors) that the considered system is able to produce the variety of patterns shown in Fig. 5. 


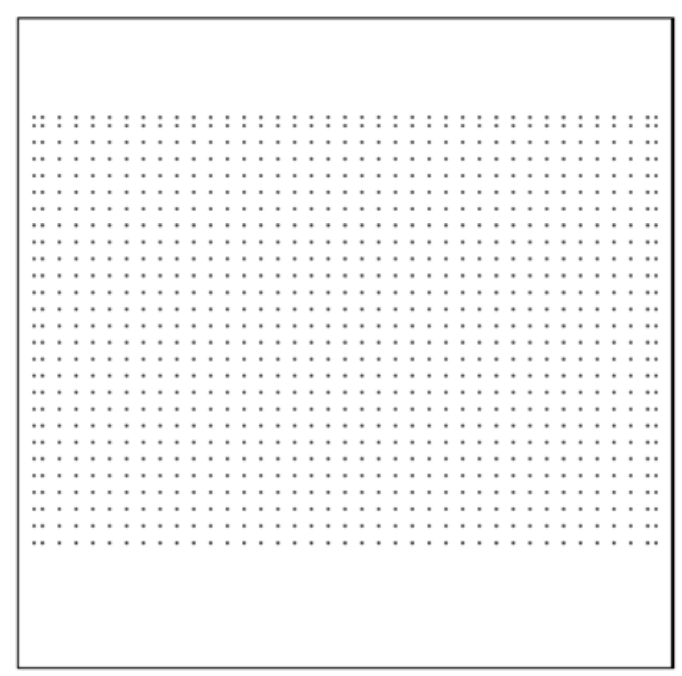

a)

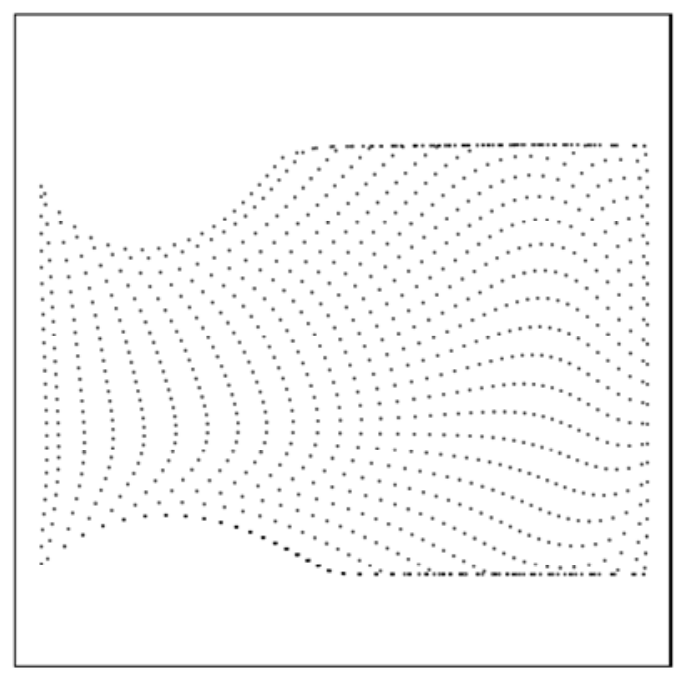

b)
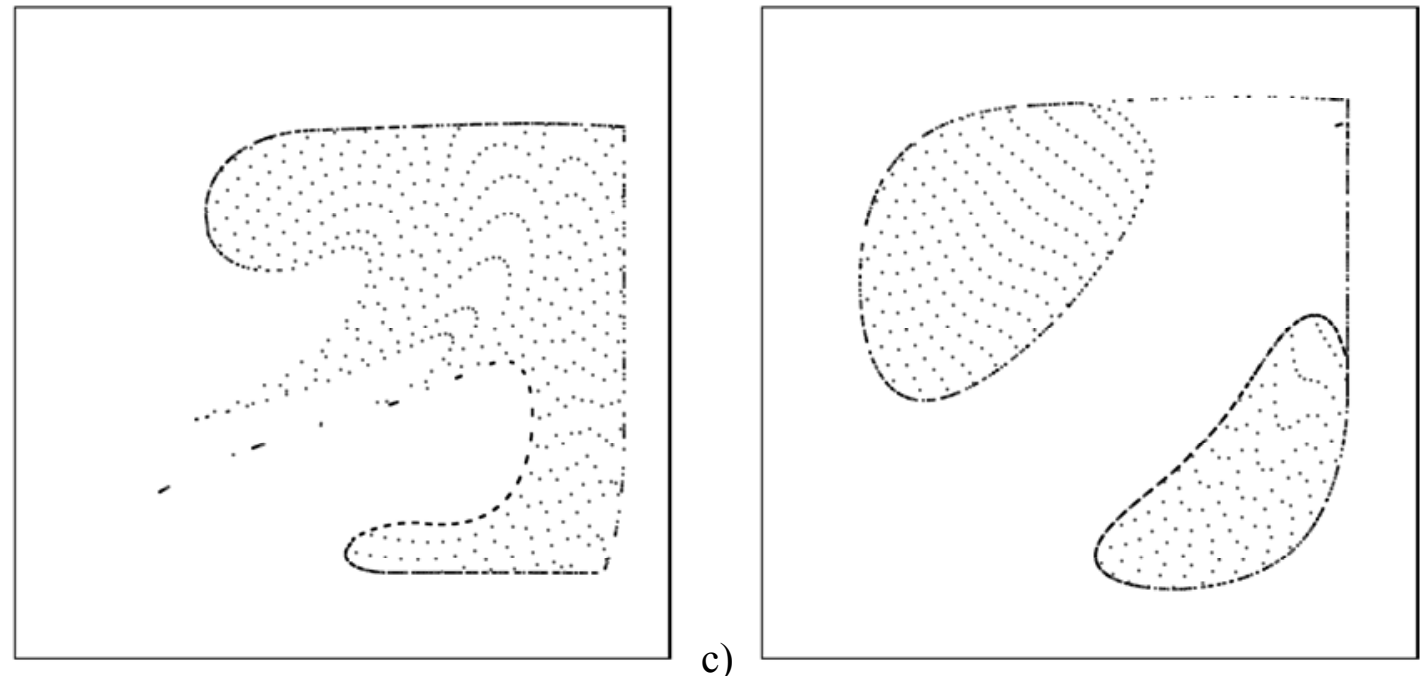

d)
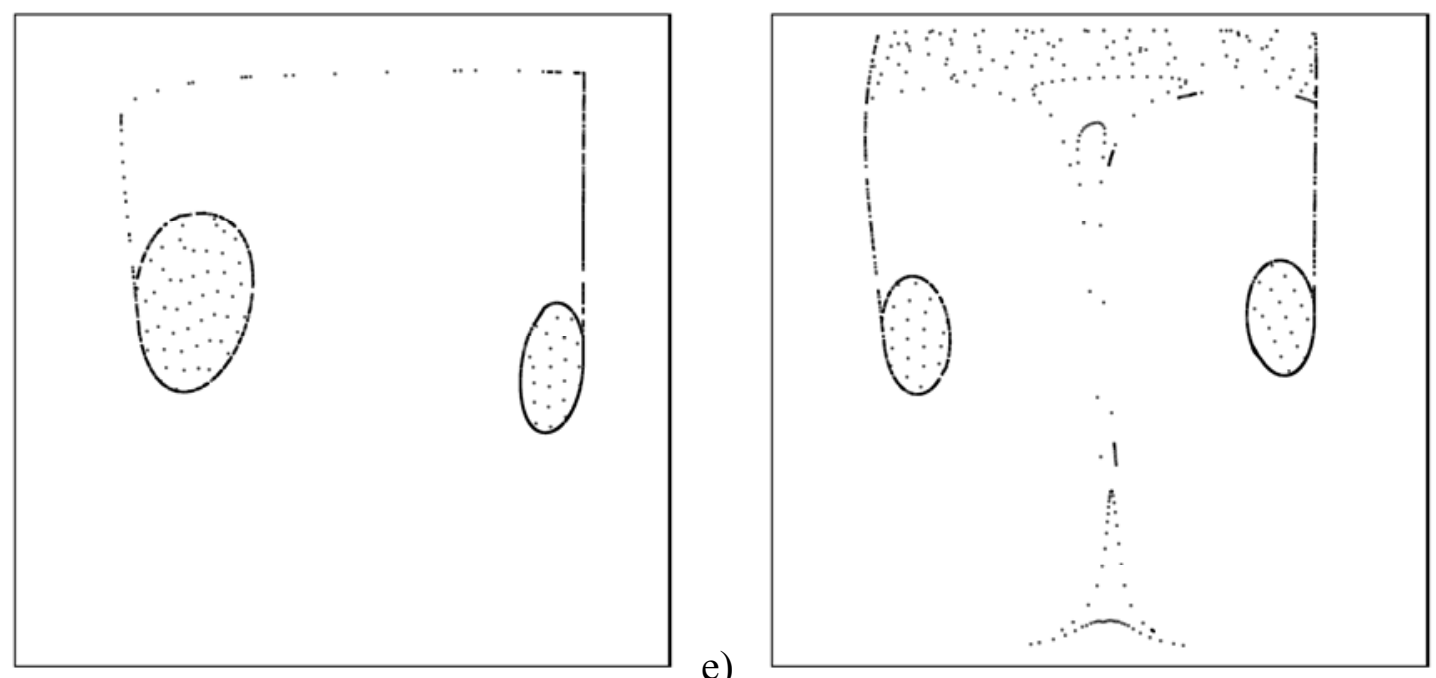

f)

Figure 5: Snapshots of particle aggregates in the section $\mathrm{y}=0$ (xz plane) as a function of the inclination angle $\varphi\left(\phi=0, \mathrm{t}=5 \times 10^{-1}\right)$ : a) $\varphi=90^{\circ}$, b) $\varphi=85^{\circ}$, c) $\varphi=67.5^{\circ}$, d) $\varphi=45^{\circ}$, e) $\varphi=22.5^{\circ}$, f) $\varphi=0^{\circ}$. 
As the reader will easily realize, the most striking feature of the particle dynamics in all cases is that the regions of the cavity adjacent to the walls perpendicular to the temperature gradient tend to become depleted. Both the morphological and topological properties of such patterns, however, change significantly when the angle $\varphi$ is varied.

The patterning behavior can be summarized as follows: the uniform dispersion of particles attained for $\varphi=90^{\circ}$ (the aforementioned degenerate condition for which no natural convection is produced and, therefore, particles experience only the influence of vibration-induced periodic acceleration along the $\mathrm{z}$ axis, see, e.g., Lappa, 2016c) is taken over by a compact (kite-like or foil-like shaped) region for $\varphi=67.5^{\circ}$ (with all the particles encapsulated inside such area and partially accumulated along its periphery). A further decrease in $\varphi$ leads to two distinct particle attractors delimited by closed (reducible) curves, until one recovers the classical case with two mirror-symmetric (with respect to the $\mathrm{z}$ axis) "circuits" obtained by Lappa (2014b) in the limit as $\varphi \rightarrow 0^{\circ}$ (Fig. 5f).

Obviously, the simplest situation is that related to $\varphi=90^{\circ}$. As it corresponds to a degenerate state, it can be used as an archetypal example to clarify that for such circumstances only the action exerted by vibrations directly on particles should be invoked to explain the observed dynamics. In this case, indeed, it can be argued that the initially perfectly uniform distribution of particles along both the $\mathrm{x}$ and $\mathrm{z}$ axes will undergo a certain "compression" in the $\mathrm{z}$ direction to be ascribed merely to the joint action of periodic acceleration (perfectly aligned with the $z$ axis for $\varphi=90^{\circ}$ ) and the "limiting" effect exerted on particles by the sidewalls at $\mathrm{z}=-0.5$ and $\mathrm{z}=0.5$.

Although for $\varphi=85^{\circ}$, the mechanism depicted above is still operative, the influence of convection on particle transport starts to play a role. Its effects can be seen in the deformation of the upper and lower sharp boundaries separating the particle accumulation region from particle-depleted areas (the clear fluid outside). Such boundaries tend to be folded (as time passes) towards the interior. This process leads in the initial stages of evolution to the formation of a horizontal amphora-like shape in the $x z$ plane (the distribution of particles results in a shape that bulges out after $x=0$ and necks in before it, Fig. 5b).

Before going further with the discussion and the interpretation of the results, at this stage we should recall once again that while the amplitude of the periodic force acting on solid particles does not depend on the angle $\varphi$ (the magnitude of this force depends only on the solid/fluid density ratio and on the amplitude and frequency of vibrations), the intensity of convection does (Fig. 6).

As an example, for $\varphi=67.5^{\circ}$ the mechanism discussed above (about the role played by convective effects in determining a folding of the boundaries delimiting the particle region) becomes so strong that it determines the collapse of the initial uniform particle distribution into the single entity shown in Fig. 5c. 


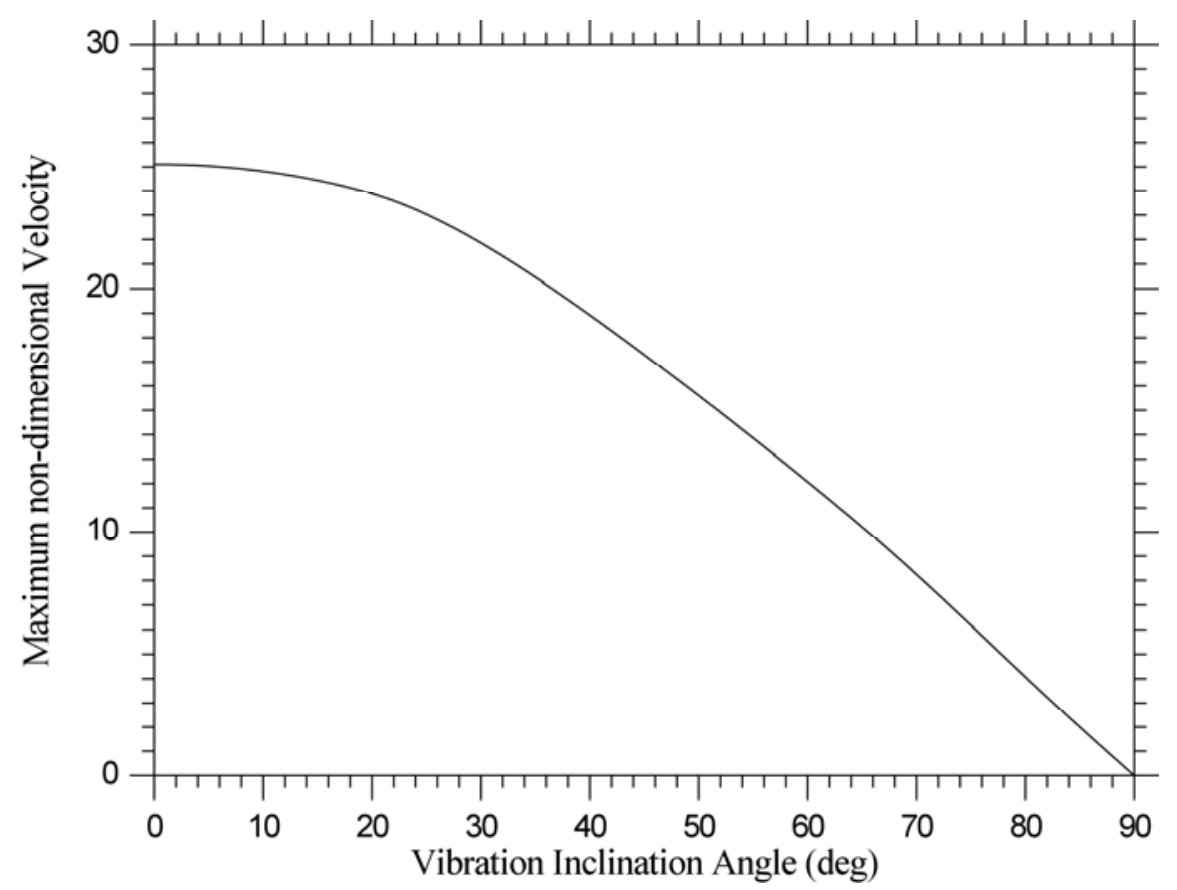

Figure 6: Maximum nondimensional velocity as a function of the $\varphi$ angle for $\phi=0$.

A fruitful strategy for obtaining insights into this apparently complex process is to take a look directly at the related stages of evolution, summarized in Fig. 7 (starting from the initial conditions). The most interesting outcome of this figure is the information it gives on the macroscopic motion of solid particles in conjunction with the constraining effect exerted on them by solid walls (such effects combine to induce accumulation of particles along specific surfaces and ensuing folding of such surfaces). The particles move essentially under the joint influence of the vibration-induced periodic force and large-scale flow of thermovibrational origin: while the former is responsible for the rhythmic displacement of each particle back and forth along the direction of imposed vibrations, the latter will be transporting the generic particle along the local direction of the large-scale flow. In particular, when a group of particles moving under the effect of the vibration-induced periodic force comes in proximity to one of the physical boundaries, particles tend all to be accumulated along the solid boundary direction (owing to the obvious impenetrability property of walls). When the periodic force changes its sign and particles are transported in the opposite direction, the above process results in the appearance of particle-dense lines in the xz plane separating the region containing particles from the pure liquid.

As time progresses and such particle-dense lines are subjected to convective transport (the largescale thermovibrational flow), they are progressively stretched and deformed producing the typical curved branches visible in the final stages of evolution in Fig. 7.

Interestingly, a further increase in time starting from Fig. $7 \mathrm{f}$ is not able to induce additional significant variations in the morphological and topological properties of this pattern. Under the effect of vibrations the pattern oscillates back and forth along the direction of vibrations as a whole, 
which indicates that the pattern-forming mechanism illustrated before has reached some asymptotic equilibrium (although it should be pointed out that, in general, the property of all particle pertaining to the pattern of moving collectively such that an illusion of solid items only applies to the pattern as such and not to individual particles, Lappa, 2014b).

Finally, we recall that for $\varphi \leq 45^{\circ}$ (Figs. 5d-5f), convective effects are even responsible for the splitting of the foil-like shape seen for $\varphi=67.5^{\circ}$ into two independent particle segregation areas. The related cause-and-effect relationships, which have been already addressed by Lappa (2014b) for $\varphi=0^{\circ}$, are not re-discussed here for the sake of brevity (the interested reader is referred to the original work for additional details).

Having finished a sketch of particle segregation phenomena in the simplified situation of vibrations contained in a plane parallel to one of the insulated walls (which produces essentially twodimensional patterns though the domain is $3 \mathrm{D}$ ), we now turn to interpreting the numerical results for the more general case of periodic accelerations arbitrarily oriented in space $(\phi \neq 0, \varphi \neq 0)$, for which the pattern is expected to develop recognizable features along the third dimension.

\subsection{Particle attractors in the general case}

As a first specific numerical example presented as a paradigm to provide inputs for an increased understanding of the underlying mechanisms when $\phi \neq 0$, we consider the case $\varphi=\phi=45^{\circ}$ (which corresponds to the peculiar condition in which vibrations are perfectly aligned with the direction $\mathrm{x}=\mathrm{y}=\mathrm{z}$ ). Different views of the related numerical results have been collected in Fig. 8 .

By comparison with Fig. $5 \mathrm{~d}$ (related to $\varphi=45^{\circ}, \phi=0^{\circ}$ ) it can be easily inferred that the inclination of vibrations in the xy plane $(\phi \neq 0)$ breaks the two-dimensional nature of the particle patterns, leading to a fully developed $3 \mathrm{D}$ configuration. Indeed, the two tubular particle structures, which for $\varphi=45^{\circ}$, $\phi=0^{\circ}$ are parallel to the $\mathrm{y}$ axis and produce (by intersection with the $\mathrm{y}=0$ plane) the two closed loops visible in Fig. $5 \mathrm{~d}$, for $\phi=45^{\circ}$ are neither parallel to this axis, nor they have the same spatial extension (one of the two structures is much shorter than the other). The emerging pattern, however, still displays some recognizable properties (essentially reflecting the symmetry of the overall vibrated system). In the specific case considered, indeed, the particle distribution is mirror symmetric with respect to the $\mathrm{y}=\mathrm{x}$ plane and one of the two attractors takes the shape of a saddlehorse paraboloid. Most remarkably, this new (heretofore unseen) structure seems to complement the set of shapes reported by Lappa (2014b), which included ellipsoids, cylinders and conical surfaces, all being geometric objects resembling the typical "quadrics" of projective geometry. 

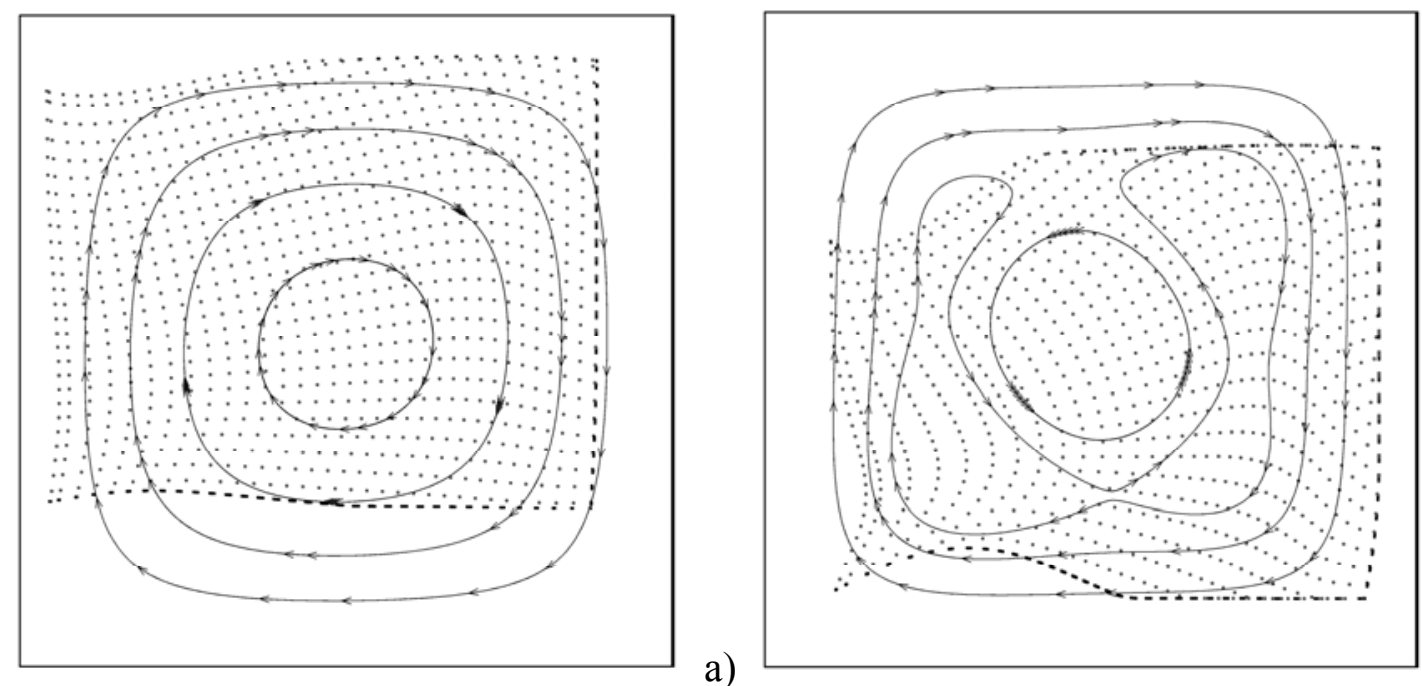

b)
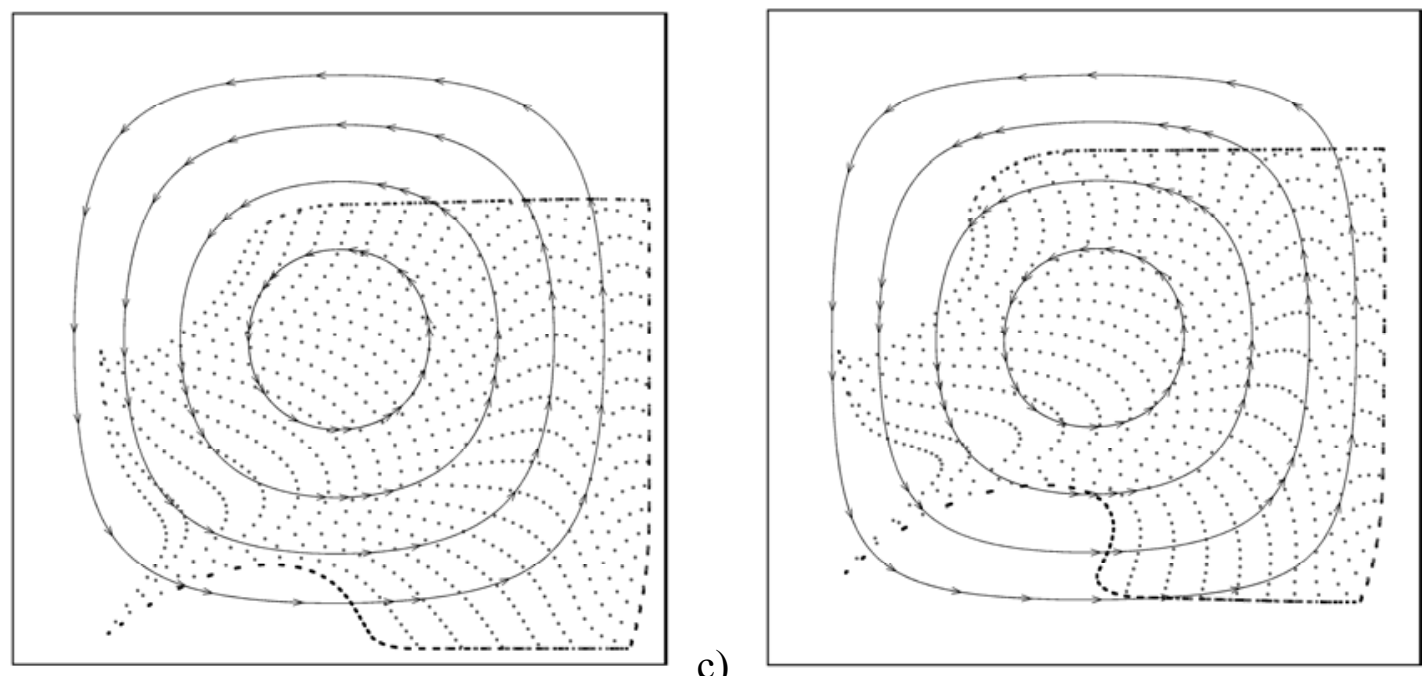

d)
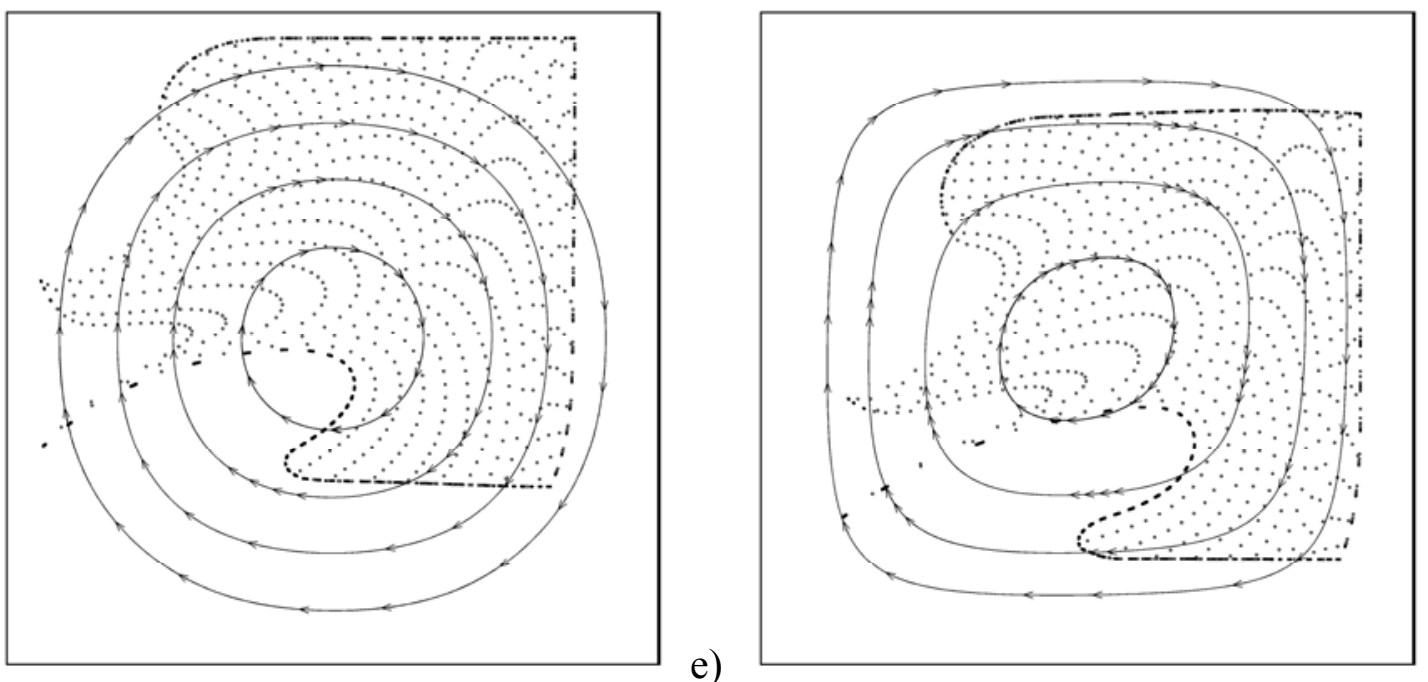

f)

Figure 7: Snapshots of particle aggregates and velocity field (in the section $y=0$ ) as a function of non-dimensional time $\left(\phi=0^{\circ}, \varphi=67.5^{\circ}\right)$ : a) $\left.\left.\left.\mathrm{t}=3 \times 10^{-2}, \mathrm{~b}\right) \mathrm{t}=9 \times 10^{-2}, \mathrm{c}\right) \mathrm{t}=1.5 \times 10^{-1}, \mathrm{~d}\right) \mathrm{t}=2.1 \times 10^{-1}$, e) $\left.\mathrm{t}=2.7 \times 10^{-1}, \mathrm{f}\right) \mathrm{t}=3.3 \times 10^{-1}$. 


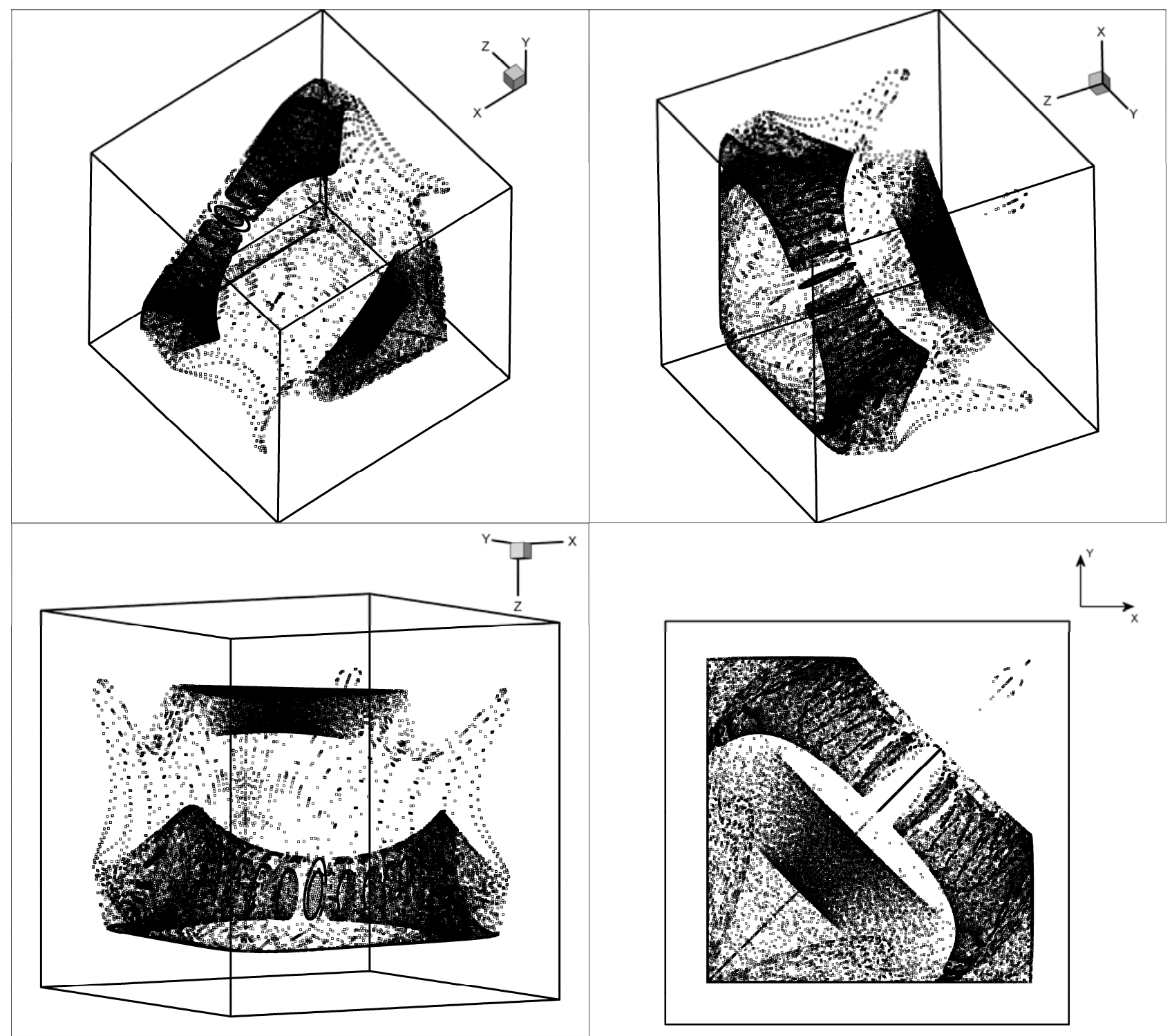

Figure 8: Particle aggregates $\left(\varphi=45^{\circ}, \phi=45^{\circ}\right.$, different views at $\left.\mathrm{t} \cong 8 \times 10^{-1}\right)$. Particles initially dispersed in the fluid collect into a seemingly rigid structure that apparently moves as a unit under the effect of vibrations.

If the angle $\varphi$ is increased to $67.5^{\circ}$ (while keeping $\phi=45^{\circ}$ ), as shown in Fig. 9, the symmetry with respect to the $\mathrm{x}=\mathrm{y}$ plane is retained. The particulate structure, however, collapses into a single compact region as we have already seen in the case $\phi=0^{\circ}$ (compare Fig. 9a and 5c), which leads to the general conclusion that the tendency of the particles to split into separate agglomerates depends essentially on the component of vibrations acting in a direction parallel to the temperature gradient. In other words, a critical $\varphi^{*}$ exists such that if $\varphi<\varphi^{*}$, the particles organize into two threedimensional substructures, whereas for $\varphi>\varphi^{*}$ the pattern formed by solid matter behaves as an apparently single unit. 


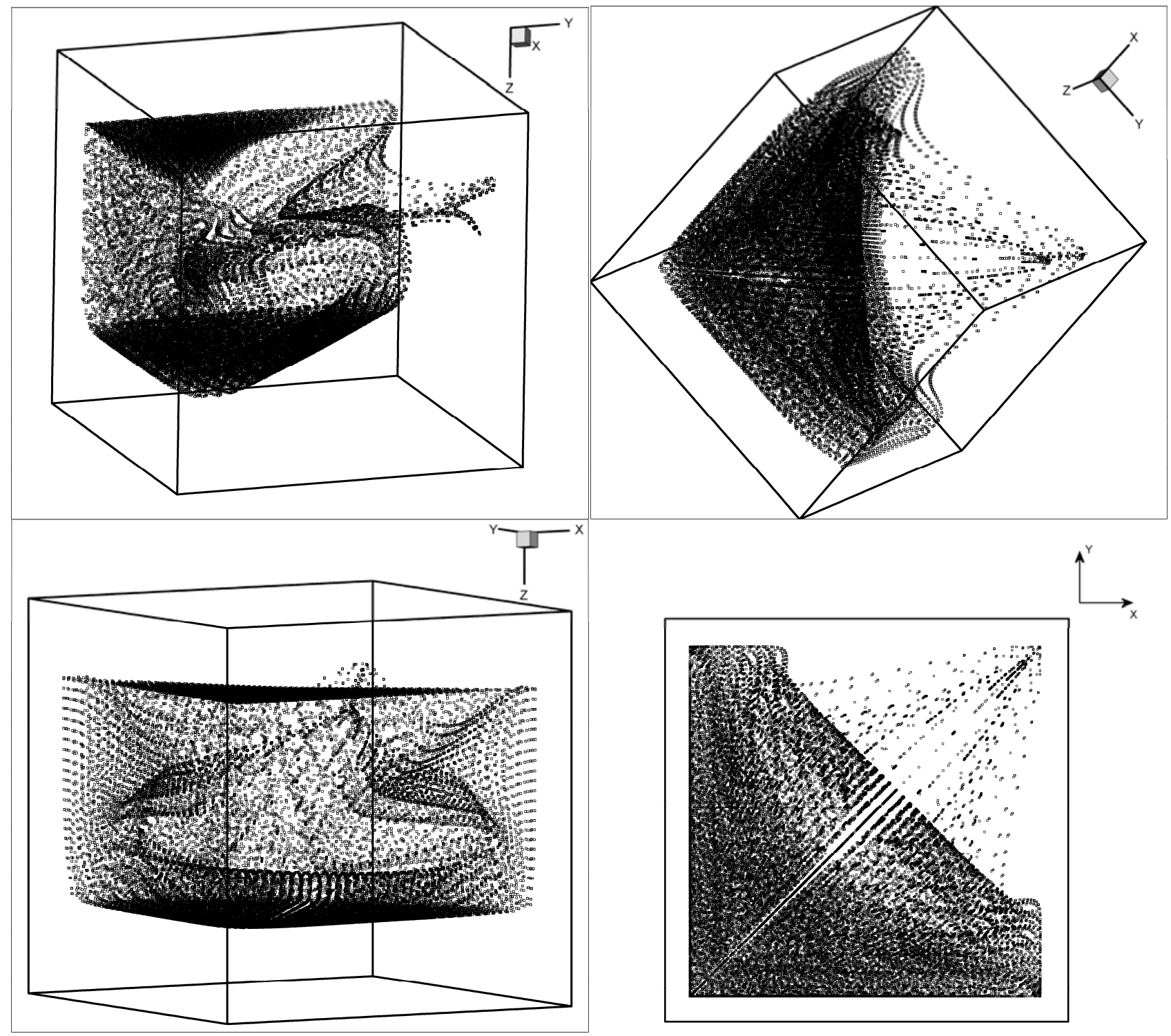

Figure 9: Particle aggregates $\left(\varphi=67.5^{\circ}, \phi=45^{\circ}\right.$, different views at $\left.\cong \cong 8 \times 10^{-1}\right)$.

This trend is confirmed by the results shown in Fig. $10\left(\varphi=45^{\circ}, \phi=22.5^{\circ}\right)$, where two distinct tubular particle structures can be distinguished again. Such patterns clearly display the "amphora-like" shape (whose formation mechanism has been already discussed to a certain extent in Sect. 3.1, see Figs. $7 \mathrm{a}$ and $\mathrm{b}$ ) and are inclined by a certain angle with respect to the $\mathrm{y}$ axis; however, the $\mathrm{y}=\mathrm{x}$ symmetry is no longer attained. This proves that while the multiplicity of accumulation regions is a function of $\varphi$, eventual symmetries in planes perpendicular to the imposed temperature gradient are possible only for specific values of $\phi$. 


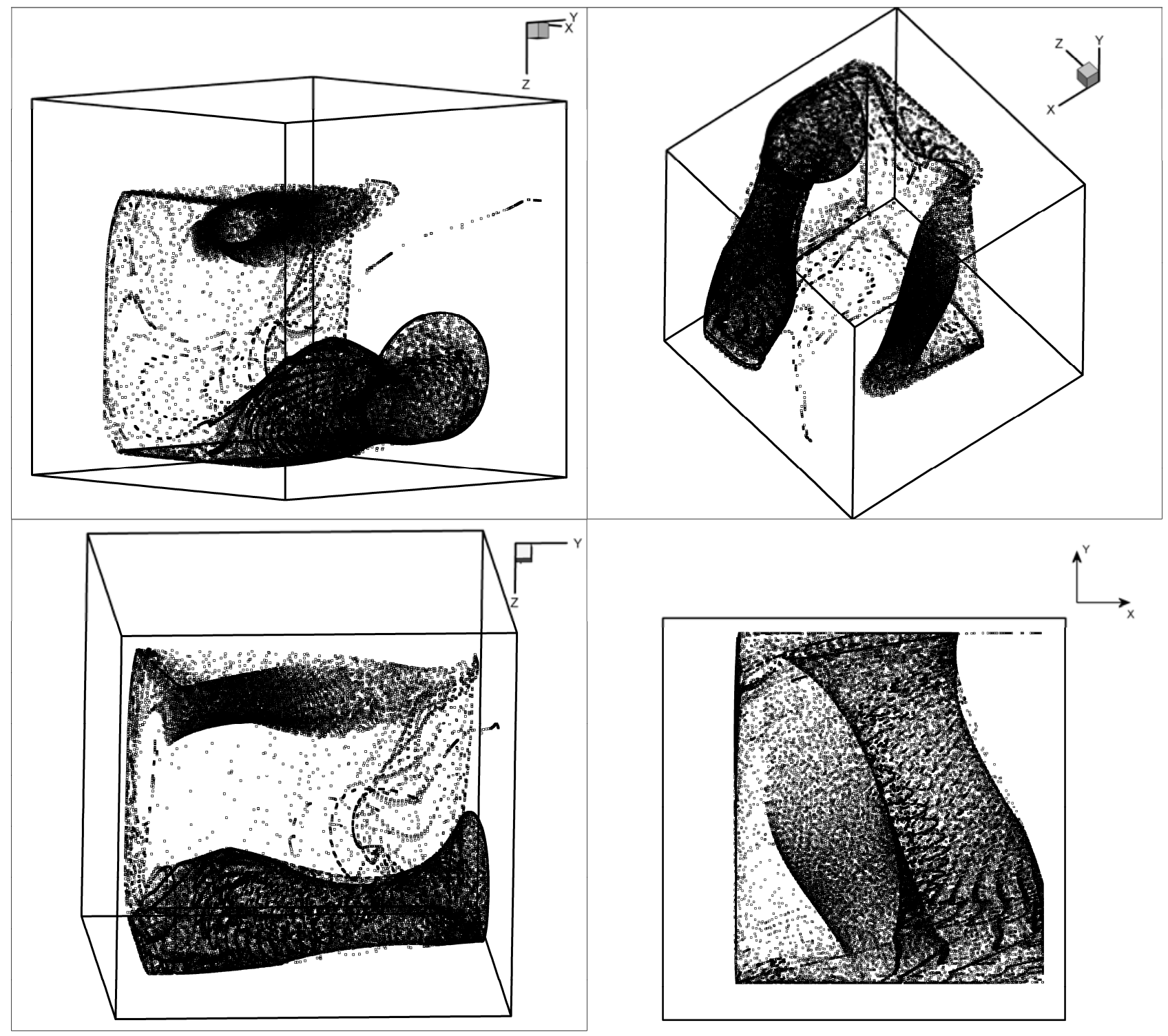

Figure 10: Particle aggregates $\left(\varphi=45^{\circ}, \phi=22.5^{\circ}\right.$, different views at $\left.\mathrm{t} \cong 8 \times 10^{-1}\right)$.

\section{Conclusions}

In this article, our focus has been on using the capabilities of our computational platform to highlight some new physical effects of particle spreading and clustering produced by the application of "vibrational forcing" to a non-isothermal fluid.

The genesis of the so-called particle "attractors" has been explored by formally allowing the direction of the imposed acceleration to cover a relatively wide range of directions in the threedimensional space. This problem, which hides a competition of complex and diverse physical processes that operate on very different spatial and temporal scales, has been analysed here from both the traditional coarse-grained macroscopic (i.e. continuum) perspective and from a finegrained micro-mechanical level in which the inertial and viscous forces acting on each single solid particle have been taken into account. 
By careful (step-by-step) comparison of the stages of evolution displayed by the vibrational flow and associated particulate structures it has been discerned that the key factor for the formation of the pattern is the mechanism produced by the "triadic" relationships among the instantaneous intensity (or strength) of oscillatory convection, the role played by solid boundaries in constraining particles and the acceleration-induced particle time-displacements (whose nonlinear effects, together with those induced by the finite size of the enclosure, accumulate over time). Such relationships are controlled or "mediated" by various symmetries and anisotropies at play in the considered system (geometry, direction of the temperature gradient, emerging flow structure, and direction of vibrations).

The multiplicity of such attractors was found to be always "two" for all circumstances examined in past work (Lappa, 2014b) regardless of the specific vibrations frequencies and amplitudes considered. Two distinct stable particle structures were observed in all cases. The number of distinct attractors coexisting in the physical space was found to be still 2 in the more recent study by Lappa (2016) though a completely different geometry was considered (a cylindrical enclosure). This led us to the wrong conclusion that the multiplicity of this category of particle attractors could be a universal number independent of the specific conditions considered. With the present study we could realize that the above conjecture was absolutely wrong. The two distinct structures can undergo "coalescence" in some cases and exist as a single attractor over a relatively wide range of conditions.

Moreover, the inclination of vibrations with respect to the insulated wall can produce attractors with new symmetric or "asymmetric" shapes not observed in the two aforementioned earlier studies, such as an amphora-like structure for $\left(\varphi=45^{\circ}, \phi=22.5^{\circ}\right.$ or a saddle-horse paraboloid for $\varphi=45^{\circ}$, $\phi=45^{\circ}$. Along these lines, the present parametric simulations have shown that, in general, while the multiplicity of accumulation regions is a function of the inclination of vibrations with respect to the temperature gradient, the 3D breakdown in the symmetry of solutions depends essentially on the inclination of vibrations with respect to the insulated sidewalls.

The identification of this new family of particle "attractors" for relatively small values of the Gershuni number, together with the related intrinsic multiplicity and sensitivity to "ambient" factors, is of significant conceptual significance as it opens new fascinating (heretofore unexplored) perspectives in the study of thermovibrational convection in combination with particle clustering phenomena and related theories for pattern formation. Because the underlying mechanism for particle accumulation does not require any kind of particle (hydrodynamic) interactions or attractive forces, the only necessary prerequisites being the existence of a temperature gradient and some inertial "disturbances" (in the form of a cyclic forcing), these dynamics might play a role in a variety of fields. In particular, vibrations appear to be a very interesting alternative for the "control" of advanced multiphase thermal processes, because, unlike other strategies, they do not require the fluid or the dispersed matter to be electrically conductive (as is the case for magnetic fields). Future work shall be devoted to study particle pattern formation in hybrid thermocapillarythermovibrational or thermocapillary-thermogravitational flows. 


\section{References}

[-] Biringen S. and Danabasoglu G., (1990), Computation of convective flows with gravity modulation in rectangular cavities, $J$. Thermophys., 4, 357-365.

[-] Busse F.H. and Clever R.M., (1992), Three-dimensional convection in an inclined layer heated from below, J. Eng. Math., 26(1): 1-19.

[-] Busse F.H. and Clever R.M., (2000), Bursts in inclined layer convection, Phys. Fluids, 12(8): 2137-2140.

[-] Castellanos A., (2005), The relationship between attractive interparticle forces and bulk behavior in dry and uncharged fine powders, Advances in Physics, 54(4), 263-376.

[-] Chen Y. and Pearlstein A.J., (1989), Stability of free-convection flows of variable-viscosity fluids in vertical and inclined slots, J. Fluid Mech., 198: 513-541.

[-] Coimbra C.F.M., L'Esperance D., Lambert R.A., (2004), An experimental study on stationary history effects in high-frequency stokes flows, J. Fluid Mech. 504, 353-363.

[-] Coimbra F.M. and Rangel R.H. (2001), Spherical particle motion in harmonic stokes flows, AIAA J, 39(9), 1673-1682.

[-] Delgado-Buscalioni R. and Crespo del Arco E., (1999), Stability of thermally driven shear flows in long inclined cavities with end-to-end temperature gradient, Int. J. Heat Mass Transfer., 42: 2811-2822.

[-] Delgado-Buscalioni R. and Crespo del Arco E., (2001), Flow and heat transfer regimes in inclined differentially heated cavities, Int. J. Heat Mass Transfer, 44: 1947-1962.

[-] Delgado-Buscalioni R., Crespo del Arco E. And Bontoux P., (2001b), Flow transitions of a lowPrandtl-number fluid in an inclined 3D cavity, Eur. J. Mech. B/Fluids, 329: 1-17.

[-] Delgado-Buscalioni R., Crespo del Arco E., Bontoux P., Ouazzani J., (1998), Convection and instabilities in differentially heated inclined shallow rectangular boxes, Compte Rend. Acad. Sci.. II B (Mechanics-Physics-Astronomy), 326(11): 711-718.

[-] Demin V., Gershuni G., Verkholantsev I., (1996), Mechanical quasi-equilibrium and thermovibrational convective instability in an inclined fluid layer, Int. J. Heat Mass Transfer, 39(9): 1979-1991.

[-] Demin V.A., (2005), Vibrational Convection in an Inclined Fluid Layer Heated from Below, Fluid Dynamics, 40(6): 865-874.

[-] Devenish B. J., Bartello P., Brenguier J-L, Collins L. R., Grabowski W. W., Ijzermans R. H. A., Malinowski S. P., Reeks M. W., Vassilicos J. C., Wang L-P., and Warhaft Z., (2012), Droplet growth in warm turbulent clouds, Quarterly Journal of the Royal Meteorological Society, 138 (667), 1401-1429.

[-] Ellison J., Ahmadi G., Regel L. and Wilcox W., (1995), Particle motion in a liquid under g-jitter excitation, Microgravity Sci. and Tech., 8, 140- 147.

[-] Fletcher C.A.J., (1991), Computational techniques for fluid-dynamics, (Springer Verlag, Berlin, 1991).

[-] Fujimura K. and Kelly R.E., (1993), Mixed mode convection in an inclined slot, J. Fluid Mech., 246: 545-568.

[-] Gershuni G.Z. and Demin V.A., (1998), Thermovibrational convective instability of mechanical equilibrium of an inclined fluid layer, Fluid Dynamics, 33(1): 5-11. Translated from Izvestiya Rossiiskoi Akademii Nauk, Mekhanika Zhidkosti i Gaza, No. 1, pp. 8-15, January-February, 1998. 
[-] Gotoda M., Melnikov D. E., Ueno I., and Shevtsova V., (2016), Experimental study on dynamics of coherent structures formed by inertial solid, particles in three-dimensional periodic flows, Chaos, 26, 073106 (11 pages)

[-] Gotoda M., Sano T., Kaneko T., and Ueno I., (2015), Evaluation of existence region and formation time of particle accumulation structure (PAS) in half-zone liquid bridge, Eur. Phys. J. Special Topics, 224, 299.

[-] Hassan S., Lyubimova T.P., Lyubimov D.V., Kawaji M., (2006), Motion of a sphere suspended in a vibrating liquid-filled container, J. Appl. Mech., 73, 72-78.

[-] Hirata K., Sasaki T., Tanigawa H., (2001), Vibrational effects on convection in a square cavity at zero gravity, J. Fluid Mech., 445, 327-344.

[-] Hjelmfelt A. T., and Mockros L. F., (1966), Motion of Discrete Particles in a Turbulent Fluid, Appl. Sci. Res., 16(1), 149-161.

[-] Houghton G., (1961), The behavior of particles in a sinusoidal vector field, Proc. Roy. Soc. A 272, 33-43.

[-] Ivanova A.A., Kozlov V.G. and Kuzaev A.F., (2005), Vibrational lift force acting on a body in a fluid near a solid surface. Dokladi RAN, vol. 402 (4), pp. 1-4 (Translated: Doklady Physics, vol. 50 (6), pp. 311-314),

[-] Kozlov V.G., Ivanova A.A. and Evesque P., (2006), Block stratification of sedimenting granular matter in a vessel due to vertical vibration, Fluid Dyn. Mater. Process., 2(3), 203-210.

[-] Kozlov V.G., Kozlov N.V., Subbotin S.V., (2017), Steady flows in rotating spherical cavity excited by multi-frequency oscillations of free inner core, Acta Astronautica, 130: 43-51

[-] Kuhlmann H.C., et al.., (2014), The JEREMI-Project on thermocapillary convection in liquid bridges. Part A: Overview of Particle Accumulation Structures, Fluid Dyn. Mater. Process., 10 (1), $1-36$.

[-] Lan C.W. and Kou S., (1991), Floating-zone crystal growth with a heated and immersed shaper - experiments, J. Cryst. Growth, 108(3-4), 541-548.

[-] Langbein D., (1991), Motion of Ensembles of Spherical Particles in a Fluid Due to g-jitter, Adv. Space Res., 11(7), 189-196.

[-] Lappa M., (2013a), Assessment of the role of axial vorticity in the formation of Particle Accumulation Structures (PAS) in supercritical Marangoni and hybrid thermocapillary-rotationdriven flows, Phys. Fluids, 25(1) 012101 (11 pages).

[-] Lappa M., (2013b), On the Existence and Multiplicity of One-dimensional Solid Particle Attractors in Time-dependent Rayleigh-Bénard Convection, Chaos, 23(1), 013105 (9 pages).

[-] Lappa M., (2013c), On the variety of particle accumulation structures under the effect of gjitters, J. Fluid Mech., 726: 160-195

[-] Lappa M., (2014a), Stationary Solid Particle Attractors in Standing Waves, Phys. Fluids, 26(1), 013305 (12 pages).

[-] Lappa M., (2014b), The patterning behavior and accumulation of spherical particles in a vibrated non-isothermal liquid, Phys. Fluids , 26(9), 093301 (22 pages).

[-] Lappa M., (2016a), On the nature, formation and diversity of particulate coherent structures in Microgravity Conditions and their relevance to materials science and problems of Astrophysical interest, Geophysical and Astrophysical Fluid Dynamics, 110(4): 348-386.

[-] Lappa M., (2016b), Control of convection patterning and intensity in shallow cavities by harmonic vibrations, Microgravity Science \& Technology , 28(1), 29-39. 
[-] Lappa M., (2016c), A numerical study into the morphology and formation mechanisms of threedimensional particle structures in vibrated cylindrical cavities with various heating conditions, Physical Review Fluids, 1(6), 064203 (25 pages).

[-] Lappa M., (2007a), Secondary and oscillatory gravitational instabilities in canonical threedimensional models of crystal growth from the melt, Part2: Lateral heating and the Hadley circulation, Comptes Rendus Mécanique, 335(5-6): 261-268.

[-] Lappa M., (2007b), Secondary and oscillatory gravitational instabilities in canonical threedimensional models of crystal growth from the melt, Part1: Rayleigh-Bènard systems, Comptes Rendus Mécanique, 335(5-6): 253-260.

[-] Lappa M., (2011), "Some considerations about the symmetry and evolution of chaotic RayleighBénard convection: The flywheel mechanism and the "wind" of turbulence", Comptes Rendus Mécanique, 339: 563-572.

[-] Lappa M., Carotenuto L., (2003), Effect of convective disturbances induced by g-jitter on the periodic precipitation of lysozyme, Microgravity Sci. Tech., XIV/2, 41-56.

[-] Love S.G., Pettit D.R., and Messenger S.R., (2014), Particle aggregation in microgravity: Informal experiments on the International Space Station, Meteoritics \& Planetary Science, 1-8 (2014) doi: 10.1111/maps.12286

[-] Maxey M.R., Patel B.K., Chang E.J., Wang L.-P., (1997), Simulations of dispersed turbulent multiphase flow, Fluid Dyn. Res., 20(1-6), 143-156.

[-] Mei R., Lawrence J., and Adrian J., (1991), Unsteady Drag on a Sphere at Finite Reynolds Number With Small Fluctuations in the Free-Stream Velocity, J. Fluid Mech., 233, 613-631.

[-] Melnikov D.E., Pushkin D.O., Shevtsova V.M., (2013), Synchronization of finite-size particles by a traveling wave in a cylindrical flow, Phys Fluids, 25 (9), 092108

[-] Monti R., Savino R., Lappa M. (1998); "Microgravity sensitivity of typical fluid physics experiment", presented at the 17 th Microgravity Measurements Group Meeting, Cleveland, Ohio, 24-26 March 1998, published in the meeting proceedings in NASA CP-1998-208414, (23): 1-15 (ISSN: 0191-7811).

[-] Monti R., Savino R., Lappa M., (2001), On the convective disturbances induced by g-jitter on the space station, Acta Astronautica, 48 (5-12), 603-615.

[-] Nield D.A., (1994), Convection induced by an inclined temperature gradient in a shallow horizontal layer, Int. J. Heat Fluid Flow, 15(2): 157-162.

[-] Pushkin D., Melnikov D., Shevtsova V., (2011), Ordering of Small Particles in OneDimensional Coherent Structures by Time-Periodic Flows, Phys. Rev. Lett., 106, 234501.

[-] Savino R., Lappa M., (2003), Assessment of the thermovibrational theory: application to g-jitter on the Space-station, Journal of Spacecraft and Rockets, 40 (2), 201-210.

[-] Schwabe D. and Mizev A.I., (2011), Particles of different density in thermocapillary liquid bridges under the action of travelling and standing hydrothermal waves, Eur. Phys. J. Special Topics, 192, 13-27

[-] Schwabe D., Tanaka S., Mizev A. and Kawamura H., (2006), Particle accumulation structures in time-dependent thermocapillary flow in a liquid bridge under microgravity, Microgravity Sci. Technol. 18, 117.

[-] Shklyaev S.V., (2001), Stability of Vibroconvective Flow in an Inclined Layer with Respect to Three-Dimensional Perturbations, Fluid Dynamics, 36(5): 682-690. 
[-] Smorodin B.L., (2003), Stability of Thermal Vibrational Flow in an Inclined Liquid Layer Against Finite Frequency Vibrations, J. Appl. Mech. Tech. Phys., 44(1): 44-51.

[-] Tagawa, Y., Martinez-Mercado, J., Prakash, V.N., Calzavarini, E., Sun, C., and Lohse, D., 2012, Three-dimensional Lagrangian Voronoi analysis for clustering of particles and bubbles in turbulence, J. Fluid Mech., 693, 201-215.

[-] Thomson J.R., Casademunt J., Drolet F. and Vinals J., (1997), Coarsening of solid-liquid mixtures in a random acceleration field, Phys Fluids, 9(5), 1336-1343.

[-] Trulsson M, Andreotti B. and Claudin P., (2012), Transition from the viscous to inertial regime in dense suspensions. Phys. Rev. Lett. 109 (11), 118305.

[-] Ueno I., Abe Y., Noguchi K., and Kawamura H., (2008), Dynamic particle accumulation structure (PAS) in half-zone liquid bridge - Reconstruction of particle motion by 3-D PTV, Adv. Space Res., 41, 2145.

[-] Wilkinson M., Mehlig B., Ostlund S.and Duncan K. P., (2007), Unmixing in random flows, Phys. Fluids, 19, 113303. 